\title{
Hacia una Metateoría Constructivista \\ Cognitiva de la Psicoterapia
}

\section{Toward a Constructive Cognitive Metatheory in Psychotherapy}

\author{
Juan Yáñez M. ${ }^{1}$, Patricio Gaete ${ }^{2}$, Tamara Harcha ${ }^{2}$, \\ Walter Kühne $\mathrm{C}^{2}$., Victoria Leiva $\mathrm{T}^{2}$., Paula Vergara $\mathrm{H}^{2}$.
}

\begin{abstract}
Resumen
El presente artículo revisa el desarrollo de la metateoría del modelo psicoterapéutico cognitivo, desde sus inicios conductuales, pasando por el modelo cognitivo tradicional hasta el constructivismo. En las bases conceptuales del modelo se revisa el desarrollo del concepto de realidad, sujeto, la concepción de conocimiento y el concepto de sí mismo. Posteriormente se explicita una metateoría Constructivista Cognitiva sobre la cual fundar la práctica clínica cognitiva.
\end{abstract}

Palabras clave: si mismo, cognitivo, constructivismo, conocimiento, sujeto.

\section{Abstract}

The present paper revises the development of the metatheory of psychotherapeutic cognitive model, from its behavioral beginnings, passing by the traditional cognitive approach, to the constructivism. The development of the concept of reality, individual conception of knowledge and the concept of self, is revised in the conceptual bases of the model. Afterwards, a Constructivist Cognitive metatheory over which we can base the clinical cognitive practice, is explained.

Key words: self, cognitive, constructivism, knowledge, individual. 


\section{Introducción}

El equipo académico de Psicología Clínica Cognitiva del Centro de Psicología Aplicada de la Universidad de Chile (CAPs), se ha propuesto delimitar un marco conceptual y aplicado dentro del enfoque Cognitivo, que tiene la intención de hacer más eficiente la atención clínica prestada a la comunidad y al mismo tiempo aportar conocimientos e información relevante a través de la investigación clínica y que se expresen además en la docencia Universitaria especializada.

La orientación cognitiva ha sufrido cambios epistemológicos importantes en el último tiempo; se advierte una creciente tendencia del modelo a hacer inclusivos fenómenos cada vez más complejos, pero que al mismo tiempo caracterizan más propiamente tal el comportamiento humano, es decir son más representativos del sujeto. Ello ha implicado necesariamente una complejización y diversificación de las teorías, los conceptos y de la práctica clínica cognitiva, lo que hace necesario delimitar con precisión un marco conceptual específico que sea posible de aplicar en un contexto de atención institucional universitaria como es el caso del CAPs .

Es precisamente a partir de estos cambios epistemológicos donde la ciencia reformula, a través de "saltos", conceptos básicos tales como realidad, conocimiento y relación sujeto/objeto. Queremos ahondar en este sentido, ya que nos interesa mostrar algunos quiebres disciplinarios que, en definitiva, nos permitirán situar nuestro paradigma.

Al respecto, Thomas Kuhn en su "Estructura de las Revoluciones Científicas (1962)" menciona como la ciencia avanza a través de crisis, es decir, a "saltos" y/o revoluciones que se consideran períodos de ciencia "anormal", pues el autor designa como ciencia normal, a aquellos períodos de calma y producción dentro de determinados supuestos y parámetros que no son cuestionados por la comunidad científica. En momentos de ciencia anormal entonces, se fragmenta la matriz disciplinaria de una teoría, y ello ocurre a su vez, a partir de la emergencia de fenómenos complejos y recurrentes que no pueden ser explicados en su totalidad por el paradigma vigente. $\mathrm{Al}$ acumularse de manera creciente la ausencia de explicación de estos fenómenos, aumenta la presión hacia una re-conceptualización del objeto modelo, así como también hacia el método de estudio de la teoría, todo lo cual da origen a una ampliación conceptual y técnica de ella que, por supuesto, permitan explicar las mencionadas anomalías.

Este artículo tiene como objetivo proponer una metateoría Constructivista Cognitiva, que nos permita sistematizar los diversos cambios conceptuales ocurridos en el modelo. Dicha metateoría resulta esencial a la hora de dar una cierta intencionalidad y coherencia a la práctica clínica. Inicialmente abordaremos los conceptos de realidad, sujeto (y su evolución), conocimiento y sí mismo, para posteriormente enunciar nuestra propuesta paradigmática.

\section{Bases Conceptuales}

Cada paradigma se sustenta, ya sea de manera explícita o implícita, en determinadas significaciones o nociones de conceptos. Abordaremos éstas nociones con la intención de fundamentar desde el punto de vista teórico, el origen desde donde desprendemos la base conceptual del Constructivismo Cognitivo. 


\section{Evolución Epistemológica y Subjetividad}

\section{a) Momento Conductual}

A comienzos de los años 20 el psicólogo norteamericano, J. B. Watson, estableció las bases paradigmáticas respecto de la teoría y la metodología de la que sería una de las principales corrientes en psicología: el conductismo. Este movimiento, que cobraría especial importancia a partir de la década del ' 50 , es una expresión de una tradición filosófica positivista, es decir, considera la existencia de un mundo real y cognoscible que existe independiente del sujeto y que esta regido por reglas inmanentes. El conocimiento es producto de la experiencia sensorial del sujeto y su validez y objetividad depende de una correlación estricta con los principios de realidad. El método que da cuenta de la rigurosidad de esta relación es el método científico a través de la verificación empírica y lógica de las teorías sobre la realidad.

Watson recogió elementos del funcionalismo, del asociacionismo y de la psicología animal. Su postura también estuvo influida por una fuerte oposición al método introspectivo propugnado por Titchener (Marx y Hillix, 1976). La principal crítica a este método era su subjetividad y falta de rigurosidad. Es así como las principales consideraciones que tiene Watson cuando propone un nuevo modelo es que este sea cien- tífico y objetivo, "la psicología, tal como la ve el conductista, es una rama experimental puramente objetiva de las ciencias naturales" (Watson, 1913, página 158; citado en Neimeyer, R. y Mahoney, M., 1998, página 187). Para ello requiere de un objeto de estudio que sea observable y cuantificable: la conducta observable, evitando la recurrencia a constructos o mecanismos subyacentes que no sean posibles de verificar su existencia, tales como la conciencia ${ }^{3}$ o la mente. Todo lo que no sea observable no cae dentro del interés del conductismo. La idea es establecer leyes en base a la observación y evitar el uso de inferencias. Las causas del comportamiento han de ser tan observables y medibles como la conducta misma.

La relación del sujeto con la realidad se establece en base a las propuestas del asociacionismo de los cuales los conductistas heredan la ecuación : E - R. Esta ecuación se fue complejizando para finalmente entender a la conducta como función de los estímulos que la anteceden, el estado del organismo, las consecuencias que se desencadenan en base a la conducta y la relación de contingencia que vincula la conducta con esas consecuencias: $\mathrm{E}-\mathrm{O}-\mathrm{R}-\mathrm{K}-\mathrm{C}$. Es decir, consideran al comportamiento como reactivo a los estímulos y consecuencias ambientales, y básicamente aprendido (Feixas y Miró, 1995). La historia de aprendizaje y condicionamientos previos del organismo tienen un valor determinante en el resultado final del comportamiento del sujeto.

\footnotetext{
3 La conciencia es un constructo particularmente esquivo. Watson considera que no sólo es imposible a un observador externo dar un reporte de ella y medirla, sino que además es casi imposible llegar a definirla de un modo satisfactorio y que no sea objetable. Mente y conciencia caen dentro de la categoría de las llamadas variables mediacionales, es decir aquellos factores que median entre el estímulo y la respuesta. Estas pueden ser de dos tipos: constructos hipotéticos, si son observables o potencialmente observables (aunque no sean observadas de hecho) y variables intervinientes, si no son observadas ni tampoco son potencialmente observables (Mahoney, 1983). Siguiendo este planteamiento los factores biológicos y genéticos son observados o al menos potencialmente observables y por lo tanto pueden tener valor explicativo y estar insertos dentro de una explicación científica. Pero la conciencia y la mente no son observables, ni siquiera es posible definirlos operacionalmente de manera satisfactoria, por lo que corresponden a variables intervinientes y no tienen cabida dentro de este modelo con pretensiones positivistas de ser científico. Al respecto Skinner plantea "la mente es lo que el cuerpo hace" (1987, página 784; citado en Neimeyer, R. y Mahoney, M., 1998, página 187), es decir, no necesitamos apelar al concepto de mente para explicar la conducta.
} 
En base a las premisas ya desarrolladas del paradigma conductual podemos concluir la concepción de sujeto que se desprende de esta tradición psicológica. El conductismo plantea al ser humano como un sujeto mecánico. Es decir, un sujeto que actúa con una reciprocidad automática ante las demandas medio ambientales y su determinación estructural biológica. Funciona como un mecanismo que responde a los factores ambientales de manera probabilística en términos de acción y reacción. Esto es, ante la presencia de ciertos estímulos y/o consecuencias, aumenta o disminuye la probabilidad de ejecución de una conducta determinada. De aquí que sea posible afirmar qué es respondiente y operante ${ }^{4}$. La aproximación conductista no explica al ser humano utilizando argumentos mentalistas $y$, mediacionales o subyacentes, sino como un sujeto reactivo ante las demandas del medio. Según las palabras de Skinner: "no es un agente originador; es un locus, un punto en el que coinciden muchas condiciones genéticas y ambientales con un efecto conjunto" (1987, página 185). En conclusión, el sujeto mecánico es externo y observable, no contiene un sí mismo (Neimeyer, R. y Mahoney, M., 1998), está vacío, despojado de conciencia, sólo contiene los músculos y glándulas que necesita para responder al medio y a sus necesidades biológicas.

El paradigma conductista es válido hasta hoy, pero tuvo su apogeo en los años '50, y aunque sus fundamentos continúan siendo válidos pierde relevancia porque su objeto es insuficiente para dar cuenta de la complejidad del fenómeno de estudio de la Psicología: El Hombre. La acumulación y la presión de ideas divergentes al interior del propio paradigma van a dar origen a un reordenamiento que mantiene su adscripción a los principios del método científico como fórmula válida para producir conocimiento, pero que propugna una ampliación del objeto de estudio, incorporando aquello que sucede al interior de la "caja negra" o sea, la subjetividad, tan resistida por Watson. Este modelo es el llamado Cognitivo Clásico.

\section{b) Momento Cognitivo}

El Conductismo y el Cognitivismo, comparten la noción de realidad sustentada por la epistemología positivista que postula la existencia de una realidad inmanente, externa al sujeto, que es la fuente del conocimiento y a la cual el sujeto accede por medio de la dotación sensorial validándose mediante la verificación tanto empírica como lógica.

El cambio que se puede observar desde una matriz conceptual conductual a una cognitiva es a nivel de paradigma, es decir que la diferencia esta en el objeto de estudio: se incluyen fenómenos mediacionales que son deducidos por una racionalidad lógica ("el salto inferencial" propuesta por Mahoney, 1983), pero también en cuanto al método pertinente para acceder a ese nuevo objeto de estudio y este sería, además de la verificación empírica, la verificación lógica, que permite evaluar la rigurosidad de la construcción del pensamiento en los sujetos.

El conocimiento es el resultado de fenómenos mediacionales, que son constructos hipotéticos que dan cuenta de procesos mentales subyacentes y que determinan la conducta final, intermediando entre el estímulo y la respuesta, y que por sí sólo son capaces de explicar el comportamiento. El método de estudio de estos fenómenos mediacionales es la racionalidad y la experimentación, pos-

\footnotetext{
${ }^{4}$ La conducta respondiente es aquella que es controlada por los estímulos que la anteceden, sean estos incondicionados o condicionados, y la operante es aquella que opera en el medio ambiente y es controlado por las consecuencias que provoca el entorno, según si estas son deseadas o aversivas. Corresponde a los desarrollos experimentales de Pavlov y Skinner. (Wolman, 1986; Skinner, 1953, 1981).
} 
tulando a la lógica, la deducción y la inferencia racional como una forma válida de conocimiento científico en la Psicología. Los representantes más conocidos de este modelo teórico son Aaron Beck, Albert Ellis, Donald Meichenbaum, entre otros.

Este modelo, que es parte de la revolución cognitiva de los '70 junto con otras disciplinas científicas, propone la existencia de un Sujeto Racional que opera determinado por sus ideas en torno a sí mismo, a los otros y el mundo, donde la conducta y la emoción son el resultado de esas ideas. Estableciéndose así, una primacía de lo cognitivo en la estructura de funcionamiento de los sujetos: El "cogito ergo sum" en este modelo es perfectamente pertinente. La emoción depende de los cambios cognitivos. La historia de vida del sujeto es considerada como "referencial" a los acontecimientos actuales, haciendo una opción por un enfoque sincrónico antes que por uno diacrónico o idiográfico; los vínculos significativos tempranos, la importancia de las relaciones interpersonales en la constitución de la subjetividad, los primeros años de vida y su significación en la elaboración del sí mismo, no son abordados o válidos en este momento cognitivo.

Se propone la existencia de procesos mediacionales complejos, organizados en torno a una estructura, constituida por los esquemas cognitivos subyacentes de orden inconsciente ${ }^{5}$, los pensamientos automáticos o fugaces de orden subconsciente ${ }^{6}$ y las distorsiones cognitivas generalmente expresadas en el discurso mediante el uso de cuantificadores universales y condicionales.
Todos estos niveles estructurales se realimentan entre sí con una dinámica que le es propia y que otorga un sello característico del ser: el estilo personal del sujeto racional, tanto en su expresión normal como patológica. Es a partir de esta concepción que se puede afirmar que la diferencia esencial entre la neurosis y la psicosis es una cuestión de "criterio de realidad" o mejor de "ajuste a la realidad".

Sin embargo, la matriz conceptual cognitiva nuevamente se hace insuficiente para explicar la complejidad creciente de los fenómenos tales como: procesos afectivos y su participación en el desarrollo de la personalidad, las relaciones interpersonales y su significancia en la construcción de la subjetividad y otros. Esta presión por nuevos cambios inicia un proceso de expansión conceptual y técnico dentro de la teoría cognitiva, centrado en que un modelo teórico adecuado que permita clarificar no sólo cómo el individuo procesa la información proveniente del exterior, sino también las formas en que los procesos afectivos, cognitivos y conductuales se desarrollan a partir de la experiencia evolutiva interpersonal. Surge, entonces, el enfoque cognitivo interpersonal.

\section{c) Momento Interpersonal}

La compleja praxis del vivir humano, presiona al modelo cognitivo por un nuevo cambio. Las emociones y los afectos, la significancia de los acontecimientos de la historia de vida del sujeto, los procesos inconscientes y otros fenómenos insuficientemen-

\footnotetext{
5 Meichenbaum y Gilmore (1984, en Kühne 2000) plantean que la cognición tiene un dominio consciente y uno inconsciente. Dentro del dominio inconsciente de la cognición estarían aquellos eventos, procesos o estructuras que están activos e influyendo sobre el pensamiento, la conducta y los afectos pero que no están siendo accesibles al dominio de la conciencia del sujeto.

6 Con la noción de subconsciente nos referimos a aquellos procesos cognitivos que comparten las mismas características señaladas para los procesos inconscientes, pero que desde el punto de vista estructural, pueden ser accesible a la conciencia mediante un acto intencionado de búsqueda por parte del sujeto. Por ejemplo, en esta categoría se incluirían los pensamientos automáticos y las distorsiones cognitivas. En otras palabras, en términos operacionales hablar de inconsciente o subconsciente son equivalentes como procesos excepto a no ser por la accesibilidad de los contenidos.
} 
te abordados por el modelo cognitivo, producen un quiebre de la matriz conceptual de nivel epistemológico. El Momento Interpersonal es la evidencia de este quiebre, que luego definitivamente se expresará en el Momento Constructivista de los años $80^{\prime}-90^{\prime}$. Representantes como Safran, Mahoney, Guidano, Liotti y otros crean las bases teóricas que antecederán la emergencia del modelo constructivo, integrando información procedente de otras tradiciones teóricas, tales como la psicología experiencial ${ }^{7}$ (Perls, Rogers, Rice), la teoría del apego (Bowlby) y la teoría interpersonal (Sullivan, 1959). Es relevante señalar que a pesar de la integración de estos aportes, se mantiene la visión de una teoría cognitiva, ya que pone acento en el modo cómo las personas construyen representaciones de su experiencia (Safran y Segal, 1994).

En el Modelo Interpersonal la realidad está en constante flujo, y el orden de ésta, corresponde a la organización que el sujeto le otorga a través de estructuras cognitivas en desarrollo creciente (en este punto se revela el precedente cognitivo), producto de las relaciones interpersonales que el sujeto tiene oportunidad de establecer a lo largo de su existencia. En otras palabras, la vida psíquica de los sujetos va a constituirse en esa particular forma de ordenar la realidad tanto contextual como interpersonal. La relación del sujeto con la realidad es a través del conocimiento de ésta y que se fundamenta en estructuras cognitivas llamadas Esquemas Cognitivos Interpersonales.

El principal aporte de este enfoque radica en establecer la noción de sujeto interpersonal, cuya conducta estaría determinada por las pautas de interacción que el individuo esta- blece con personas significativas durante los primeros años de su vida y que en el presente se actualizan de modo inconsciente. El niño construye un modelo de relación con sus padres basado en las interacciones cotidianas con ellos. "Las pruebas demuestran que una vez construidos estos modelos de un padre y un sí mismo en interacción, tienden a persistir y se los da por sentado en grado tal que llegan a operar a nivel inconsciente... las pautas de interacción a las que conducen los modelos, una vez que se han vuelto habituales, generalizadas y en gran medida inconscientes, persisten en un estado más o menos no corregido e invariable incluso cuando el individuo, en años posteriores, se relaciona con personas que lo tratan de maneras totalmente diferentes a las adoptadas por sus padres cuando él era un niño..." (Bowlby 1989, pág. 152). Este modelo operativo interno puede concebirse como un esquema interpersonal que permite la predicción de nuevas interacciones sociales. Desde esta perspectiva, podría definirse el esquema interpersonal como un plan para mantener las relaciones interpersonales (Safran y Segal 1994).

El sujeto interpersonal no es un ente pasivo que recibe información proveniente de una realidad objetiva, visión del sujeto mecánico y cognitivo, sino que es un sujeto activo que construye su realidad según su propia estructura y organización, lo que habla más de su funcionamiento que de una realidad externa y objetiva. Por lo tanto, podemos decir que hay un cambio desde una epistemología positivista, que considera una realidad externa y absoluta y la posibilidad del sujeto de acceder a ella, hacia una epistemología constructivista, que considera al individuo construyendo su propia realidad.

7 Esta perspectiva concuerda con la teoría experiencial en la idea que el sujeto es el único experto en lo concerniente a su propia realidad (Perls 1973, Rice 1974 y Rogers 1986 en Safran y Segal 1994). 


\section{d) Momento Constructivista}

La realidad desde lo Constructivista es entendida como "una red de procesos pluridimencionales entrelazados, articulados simultáneamente en múltiples niveles de interacción" (Guidano, 1994). En esta idea de que la realidad sólo es posible concebirla en la propia experiencia del sujeto, se sustenta la afirmación de $\mathrm{H}$. Maturana que señala el paso desde una noción de realidad como Universo a una concepción de la realidad como Multiverso. Esta distinción se realiza teniendo en cuenta que la experiencia particular de un individuo constituye un universo propio entrelazado en una red de relaciones ordenadas en el lenguaje. Se entiende de este modo, la importancia que cobra el hecho de que la realidad sea construida en un ámbito de orden social e inexorablemente intersubjetivo. De esta manera, el sujeto constructivo asume de lleno el hecho de que cualquier observación que se realice, no puede ser separada del observador. Es así como el constructivismo, postula la realidad como la elaboración individual y colectiva de significados con respecto al sí mismo y al mundo. En este sentido, una realidad es una creación del lenguaje que sólo puede surgir como fenómeno interaccional, es decir, aparece como una actividad de orden social.

La línea argumental constructiva, que destaca las características proactivas del ser humano, la esencia tácita de sus procesos de ordenación y clasificación y por último, la inmersión de la experiencia humana en un ámbito social (lenguaje), será la nueva matriz conceptual que proveerá de una epistemología capaz de incorporar con profundidad aquellos fenómenos que resultan ser los contenidos que caracterizan más propiamente al ser humano y que permiten abordar desde una perspectiva más dinámica y genética, tanto los fenómenos "normales" como los "patológicos". Algunas de las ideas centrales del Constructivismo son:
Primero, que la "mente" es un constructor y que el "psiquismo" es el resultado de un proceso de construcción, de modo tal que los contenidos mentales no son ni innatos ni adquiridos en una relación contingente con el medio, sino que construidos. Piaget (1997) dirá al respecto, que el conocimiento es una asimilación de lo real a las estructuras previas del sujeto. Una apropiación del mundo que tiene el carácter de transformación, que corresponde a una "acción hacia", que constituye el conocimiento. Él va a dedicarse a investigar estas estructuras a priori, preexistentes a la experiencia del sujeto con la realidad. Intenta dilucidar el carácter de estas estructuras y el cómo llegan a formar parte del sujeto: la génesis de las estructuras del ser humano (Jean Piaget, 1986). Al respecto John Searle dirá: "La conciencia es una propiedad mental y, por lo tanto, física del cerebro en el sentido en que la liquidez es una propiedad de sistemas de moléculas... el hecho de que un rasgo sea mental, no implica que no sea físico; el hecho de que un rasgo sea físico no implica que no sea mental. Revisando en este momento a Descartes podríamos decir, no sólo "Pienso, luego existo" y "Soy un ser que piensa", sino también "Soy un ser pensante, luego soy un ser físico" (Searle, 1996).

Otra idea planteada por el constructivismo, y muy relacionada con la anterior, se refiere a que la mente -fruto de un proceso autoconstructivo- se convierte en el instrumento a partir del cual se construye lo real. Es decir, la realidad sólo es posible desde la experiencia. Esta tesis o argumento es muy debatida actualmente y da origen a diversas propuestas. Maturana al respecto, dirá que se debe poner la objetividad entre paréntesis y que el observador está siempre involucrado en lo que observa.

La tercera idea, reconoce la primacía funcional y estructural de los procesos abstractos (tácitos) sobre los concretos (explícitos) 
en toda experiencia emocional y consciente. Entonces, de acuerdo con el constructivismo, la experiencia humana está compuesta por dos niveles de procesamiento: Un nivel de ordenamiento holístico en términos de intuiciones perceptivas de configuraciones espacio-temporales (predominantemente tácito o inconsciente)' (Balbi, 1994, página 57), que es el nivel que Guidano llama "nivel de la experiencia inmediata". El otro nivel de la experiencia humana es de 'ordenamiento en términos de procesos secuenciales, semánticos y analíticos "predominantemente explícito o consciente" (ibídem), al que Guidano llama nivel explícito. El nivel tácito corresponde principalmente al dominio de las emociones, que es la primera forma de conocimiento tanto a nivel de desarrollo filogenético como ontogenético. Los seres humanos somos, antes que seres racionales, seres emocionales y vivimos en un mundo intersubjetivo.

Bajo esta perspectiva entonces, ya no es posible suponer una realidad ajena, independiente o universal, distinta al sujeto y por tanto única y verdadera. La realidad es construida en la interacción entre el medio que circunda a un sujeto dado (que incluye también al medio intraorganísmico) y la discriminación de este mismo organismo entre sus propias operaciones o estados (o lo que se denomina también clausura operacional del sistema nervioso). El medio y sus estímulos se transforman, de esta manera, sólo en agentes gatilladores que perturban el dominio de acción del sistema nervioso humano. Así entonces, la respuesta de cada organismo no está dada por el estímulo en sí, sino por la perturbación que éste provoca, dadas las características estructurales y organizacionales propias del organismo. Este postulado, sustentado en la biología, implica un "salto" epistemológico trascendental: ya no es posible hablar de la realidad, sino sólo podemos referirnos a una realidad particular de un sujeto particular que se genera y co-construye en la dinámica interaccional.

Desde el punto de vista Contructivista el conocimiento es entendido, por Guidano y Liotti (en Mahoney, 1988), como una construcción progresiva y jerárquica de modelos de realidad de manera tal que el sujeto acople y coordine sus experiencias a determinadas estructuras internas de conocimiento. El conocimiento entonces, ordena la relación del sujeto con el medio en un proceso unitario que implica los aspectos cognitivo, emocional y conductual. De esta manera, aparece un sujeto humano que crea teorías sobre el ambiente y sobre sí mismo, teorías a través de las cuales opera en el mundo (Weimer 1984, en Guidano 1994).

Cuando hablamos de construcciones jerárquicas, queremos destacar el carácter potencialmente transitorio de estas construcciones, es decir que éstas pueden ir aumentando su nivel de inclusión en relación al fenómeno que pretende explicar. Así, las teorías serán viables en la medida que le permitan al sujeto "la conservación de una adecuación adaptativa" (Guidano). Las teorías creadas por los sujetos son teorías que han buscado explicar y elaborar de un modo integrador y autorreferencial una mayor cantidad de experiencias y no un acercamiento a la verdad entendida de manera objetiva.

Al hablar de autoorganización, el constructivismo plantea que es justamente el sujeto el constructor de estas teorías sobre su ambiente. La llegada a nuevos niveles de conocimiento, implica un cambio en la forma de ver la realidad por parte del sujeto; esto quiere decir que existe interacción entre la actividad del sujeto y su visión de mundo. Esta visión también cambiará de acuerdo a su actividad.

El sujeto constructivo se caracteriza por ser proactivo. Esto implica que se genera a sí mismo en una dinámica que ocurre entre 
realidad y la propia organización y estructuras del sujeto. Así, ambos elementos, sujeto y realidad, se establecen en una relación $m u$ tua e indiferenciable.

La experiencia humana, ocurre en un doble nivel, explícito e implícito. El sujeto constructivo, necesita para mantener su coherencia interna, dar y darse continuas explicaciones sobre sí mismo y el mundo; ahora bien, estas explicaciones son de orden consciente. Sin embargo, este nivel explícito se encuentra subordinado a la forma única y particular de organizar su propia experiencia. Este patrón inconsciente que organiza su experienciar y lo incorpora a sus propias estructuras, es susceptible de ser conocido. Al respecto Guidano (1994) plantea la existencia de constantes experienciales denominadas Organizaciones de Significado Personal (O.S.P.), construidas a partir de el conocimiento del nivel de la explicación (cognitivo) y de la experiencia inmediata (emoción).

Como consecuencia de lo anterior, la Metateoría y la teoría clínica cognitiva, transitan desde la objetividad a la subjetividad y más aún, a la intersubjetividad, con lo cual cobran de este modo mayor relevancia fenómenos más intangibles y particulares como las relaciones, los procesos internos y las emociones entre otros.

\section{Concepto del sí Mismo}

De acuerdo con la epistemología Constructivista, la subjetividad se entiende como un Sí Mismo que opera a través de un sistema de conocimiento y autoconocimiento que le permite hacer consistente, en la explicación, la experiencia de la praxis del vivir. El sujeto entonces, se constituye en una intrincada relación entre sus experiencias vitales y la forma idiosincrásica de organizar (explicar) estas experiencias, de modo tal que le permitan progresivamente hacer aquellas explicaciones coherentes con esquemas prototípicos evolutivos. Estos esquemas progresan en complejidad y en sus posibilidades de incluir perturbaciones cada vez más discrepantes. En esta dinámica, de la necesidad de conocer el mundo y al mismo tiempo de procesarlo de una manera consistente con los propios esquemas, va conformando la subjetividad. Ésta le da un sello propio a cada individuo, le permite distinguirse de los otros y de sus múltiples formas viables de vivir, entre las cuales él es uno más.

Proponer el Sí Mismo como un sistema de conocimiento, significa referirse al efecto de la operación de una particular estructura y de su organización, cuyo dinamismo puede ser entendido como una unidad diferenciada de otra, sólo desde el lugar del observador que es perturbado por los efectos de la operación de esa unidad y quién atribuirá, en virtud de sus propias características operacionales, y no como una característica intrínseca del sistema observado, el carácter de unidad.

Por una parte, la estructura del Sí Mismo, será entendida como una disposición particular de los componentes del sistema, cuyas relaciones le permiten presentar esa particular disposición. La estructura del si mismo está compuesta en primer lugar, por esquemas de conocimiento unificados progresivamente en la relación del sujeto con la experiencia de su praxis vital. Estos esquemas le permiten adscribir significados a la experiencia y expresarlos en creencias y reglas sobre Sí Mismo, la realidad y la relación del sujeto con ésta, lo que equivale a la propuesta de Guidano (1991) relativa al "proceso continuo de reordenamiento y reconstrucción del propio sentido del Sí Mismo". El autor propone un nivel de conocimiento explícito correspondiente al "Mí" que observa, evalúa y se explica desde un punto de vista objetivo, en un nivel semántico de la experiencia. En 
esta parte de la estructura del Sí Mismo es donde se encuentran los esquemas semánticos de conocimiento que permiten sustentar una narrativa coherente con la noción de Sí Mismo que sustenta la identidad del sujeto.

Un segundo elemento de la estructura del Sí Mismo son los contenidos experienciales que a partir de las perturbaciones del medio sobre el Sí Mismo, constituyen el material vivencial para ser procesado por los esquemas de conocimiento. Es el "Yo" que experimenta momento a momento, el sentido de Sí Mismo, en la dinámica corporal, acompañada de ritmos psicofisiológicos que proporcionan continuidad a la experiencia inmediata. Dicha continuidad es expresada por Stern (en Safran y Segal, 1994) en su propuesta de cuatro constantes experienciales cruciales para mantener la coherencia interna del sentido de Sí Mismo:

1. El agente o la autoría de la acción: es la constante fundamental de la experiencia del Sí Mismo que permite aprender a manipular el mundo con fines de supervivencia y que esta compuesta por:
a) el sentido de volición que antecede a un acto motor y por el
b) sentido de predictibilidad de las con secuencias de las acciones.

2. La autocoherencia: sentido de ser una totalidad física no fragmentada, con fronteras y un centro de acción integrado es decir, un sentido unitario de Sí Mismo.

3. La autoafectividad: organización de la experiencia en diferentes estados afectivos. Corresponde a un patrón emocional característico e invariable. Esta constante es equivalente al concepto de tonalidad emotiva básica co- rrespondientes a las distintas Organizaciones de Significado Personal (Guidano, 1994).

4. La autohistoria: continuidad temporal de la organización de la experiencia. La operación de esta estructura del Sí Mismo da origen al conocimiento tácito referido a los procesos profundos de la actividad del conocimiento que abstraen las características claves de la interacción continua con la realidad.

Por otra parte, la organización de este sistema de conocimiento (Sí Mismo) se refiere a la secuencia de interacciones que le confieren al sistema sus características operacionales. En otras palabras, la modalidad particular en que los elementos del sistema interactúan entre sí. Éstas son interacciones mutuamente necesarias para la marcha de la estructura y le otorgan un sentido estable de "identidad" al sistema. La relación entre la estructura y la organización en constante interacción con las perturbaciones medio ambientales, van a determinar la dinámica particular de un organismo que las enfrenta y las hace propias a partir de la operación de su propio sistema de conocimiento.

Los aspectos discrepantes que surgen de dicha interacción, son incluidos al sistema a partir, de explicaciones parciales que dan origen a lo que en la teoría clínica denominamos "sintomas" y que constituyen aquellos contenidos que no pueden ser simbolizados, o sea agregados o integrados a los esquemas de conocimiento. Estas explicaciones parciales y discrepantes forman sistemas experienciales (síntomas) que le permiten al sistema elaborar nuevos elementos para lograr su integración, generándose de esta manera un sistema recursivo entre la experiencia y la explicación. Dicha recursividad finaliza sólo cuando el sujeto 
es capaz de simbolizar dicha información e integrarla a sus esquemas de conocimiento. Por lo tanto, esta dinámica constituye lo denominado como proceso de "cambio", de acuerdo con Guidano y Liotti (en Mahoney 1985). Este proceso es el regulador principal de la dinámica del Sí Mismo puesto que le permite al sujeto reorganizar, flexibilizar y hacer más inclusiva las perturbaciones o demandas ambientales, permitiéndole de una forma idiosincrática explicar sus experiencias.

Aquellas experiencias que pueden ser integradas a los esquemas de conocimiento constituyen el proceso de mantenimiento, el cual es esencial para el logro de una autoidentidad estable. De esta manera el proceso de mantenimiento confirma la fiabilidad de la forma que el sujeto tiene de verse y experimentarse a Sí Mismo y a la realidad (Guidano y Liotti en Mahoney, 1985). Al mismo tiempo permite sentir las discrepancias de dicha forma en determinadas circunstancias.

\section{Constructivismo Cognitivo}

Hemos realizado ya una descripción analítica de los conceptos involucrados, sin embargo queremos retomarlos en un sentido más específico.

Al hablar de Constructivismo Cognitivo, nos referimos a una propuesta teórica, que pretende enfatizar que el sujeto humano, a través de estructuras cognitivas a priori, instaladas como resultado de su particular ontogenia y filogenia, establece una relación con la realidad, cuyo resultado son sistemas de conocimientos organizados a partir de el lenguaje, que convertido en el vehículo simbólico abstracto, le posibilita la coordinación de sus experiencias internas con la comunidad de relaciones interpersonales en que esta sustentado. El hecho de organizar la realidad en un sistema lingüístico, es lo que permite al sujeto, por una parte, abrir la posibilidad de hacerla consensual, y por otra apropiarse de ella a través de construcciones progresivas coherentes con la propia organización, tanto así que puede atribuir la condición de ser " objetiva ", aún cuando no sea otra cosa que el resultado de su propio vivir.

Por otra parte, el ser humano, mientras esta en ésta dinámica constituyente (estructuras internas de conocimiento, ontogenia, filogenia, medio ambiente social y lenguaje) no solo aprehende una noción de lo real, complementariamente también adquiere una noción de sí mismo distinto de los otros, en la reciprocidad interpersonal.

La referencia a lo constructivista pretende hacer énfasis en la naturaleza activa y proactiva ${ }^{8}$ del conocimiento. $\mathrm{El}$ aspecto cognitivo hace hincapié en que estos procesos de conocimiento "son el resultado del emparejamiento continuo entre la información que llega y los esquemas contextuales que resultan del nivel tácito y explícito del conocimiento" (Guidano y Liotti en Mahoney, 1988 )

Un sentido semejante tiene la propuesta fenomenológica de Husserl (1951) en "La Filosofía como Ciencia Estricta", en el sentido de la estrictez distinta de la exactitud. Se postula la objetividad del razonamiento hipotético-deductivo respecto de aquella estrictez, que supone asumir que en la descripción de un fenómeno se incluye al suje-

\footnotetext{
8 Entenderemos proactivo como aquel proceso de conocimiento que indica una continuidad hacia delante del acto de conocer, destacándose el papel activo por parte del sujeto acerca de la realidad. Kant y Freud en sus respectivos escritos sobre el conocimiento sintético a priori, lo indican como "...la proyección de las realidades personales sobre las configuraciones externas. Los mecanismos que están involucrados en este proceso de conocimiento proactivo son realmente preontológicos, en el sentido que ponen límite a la naturaleza y al rango de la experiencia asimilable» (en Mahoney, 1998)
} 
to que observa (yo fenomenológico). El sujeto que realiza el acto de conocer (reducción fenomenológica), está ahí colocado como prioritario en la descripción, de tal manera que al final algunas de las características de la descripción del objeto, van a ser características del sujeto que observa. De esta manera, la fenomenología trata de postergar todo razonamiento causal, proponiendo un camino de conocer, vía la descripción.

Popper y Eccles (1980) dirán respecto de la construcción de lo real, que es producto de un comportamiento hipotético, ya que el sujeto humano es capaz de elaborar hipótesis, de generar una ciencia hipotéticodeductiva, es decir que es sólo una apariencia o una metáfora hipotética que "el mundo nos entre por la mirada, en realidad, el mundo nos sale por la mirada". Esta concepción corresponde a lo que se ha denominado "Teorías Motoras de la Mente", donde la mente aparece como un sistema, (con estructura y organización), proactivo y constructivo, capaz de operar respecto de su producción y su recepción, incluyendo las sensaciones básicas que subyacen a este mismo proceso.

Al respecto Michael Mahoney (1991) propone que: "el conocimiento humano es activo, anticipatorio y literalmente constructivo (de dar forma). A diferencia del retrato -más pasivo- de la mente y los procesos mentales que ofrecen las teorías no constructivistas, ésta considera la representación mental como proactiva, generadora y personificada. En cierto sentido, el constructivismo argumenta que los humanos son cocreadores de las realidades a las que responden. Esta afirmación puede provenir del pasado, de los escritos de Giambattista Vico e Immanuel Kant en el siglo XVIII; luego en los siglos XIX y XX la elaboraron de nuevos escritores como Hans Vaihinger (1911-1924), Wilhelm Wundt
(1912), Franz Brentano (1929-1981), Frederic Bartlett (1932), Jean Piaget (1970) y George Kelly (1955; ver también Maher, 1969). A causa de este retrato proactivo de la representación mental, la metateoría constructivista abarca una aproximación al conocimiento más de arriba hacia abajo que de abajo hacia arriba (Gardner, 1985; Guidano, 1987, 1991; Hayek, 1952; Mahoney, 1988, 1991; Weimer, 1977)."

Vittorio Guidano por su parte, señala que en oposición a las teorías sensoriales de la mente están las teorías motoras. "De acuerdo con la perspectiva motora, se considera a la mente como un sistema de reglas abstractas que imponen un orden relativo en la experiencia y la actividad (Weimer, 1982). Tal y como apuntaba Hayek (1978), la riqueza del mundo sensorial que experimentamos no constituye el punto de inicio del que se derivan o provienen las abstracciones mentales, sino que por el contrario, éstas son el resultado de un amplio abanico de abstracciones que la mente debe trabajar con el fin de ser capaz de experimentar tal riqueza de detalles. Lo que se dio por supuesto al explicar el funcionamiento de la mente humana - que lo principal era lo concreto y que lo abstracto parecía derivarse de él -, parece ahora ser un error de nuestra experiencia subjetiva, reflejando la complejidad y la capacidad automática de organización que la mente humana ha adquirido a lo largo de su evolución (Hayek, 1952)", (Guidano, 1994).

No podríamos sobrevivir si no fuera porque establecemos lazos y vínculos con otros seres significativos. Esto a un nivel concreto del infante que necesita de quien le proteja y alimente, y también posteriormente respecto de la necesidad de constituirse como un sí mismo, necesidad vital para la supervivencia y que sólo puede realizarse a través de la interacción con otros (Guidano, 1994). Esta interacción es realizada en un 
primer momento en la vida de los individuos a un nivel meramente emocional. Los estilos de vínculo y apego con los cuidadores, normalmente los padres, van posibilitando la ocurrencia de estados emocionales recurrentes en el niño que paulatinamente van siendo reconocidos como propios y como constitutivos de un sentido de identidad. En esa misma dinámica se va incorporando el lenguaje humano, capaz de reordenar esta experiencia emocional en un nivel semántico, que le permite comprenderla, ordenarla, expresarla o incluso simularla ante los otros. Esta dimensión explícita permite al sujeto observarse y referirse a sí mismo desde el punto de vista de los otros. Siendo las relaciones interpersonales y la calidad emocional de los vínculos tan dramáticamente importantes para nuestra especie, este nuevo nivel de ordenamiento de la experiencia nos permite reordenarla en función de la mantención de la coherencia del sí mismo y del apego con otros significativos.

En síntesis, desde el constructivismo cognitivo se considera que la experiencia humana está siendo vivenciada constantemente a través de dos niveles distintos, simultáneos e interactuantes de procesamiento de la información. Un nivel emocional, inmediato y principalmente inconsciente, y un nivel semántico y analítico principalmente consciente que se refiere a la experiencia inmediata y la reordena en función de mantener la coherencia del sí mismo.

Por lo tanto, a partir de esta revolución epistemológica que resulta del argumento constructivo, las implicancias para el modelo son amplias, fundamentales y diversas. Se puede pensar en un sujeto constructivo, que opera con dispositivos epistémicos, elaborados en la dinámica particular con el medio (deriva ontogénica $\mathrm{y}$ acoplamiento estructural para
Maturana). Estas estructuras mentales se coordinan con las perturbaciones ambientales, aunque no están determinadas por estas, van aumentando, de modo creciente y progresivo en complejidad, a partir de la instalación del lenguaje, y de la relevante condición de constituirse en un ser humano, siempre en el ámbito de una estrecha relación con un otro significativo (teoría del apego de John Bowlby (1993). La validación intersubjetiva que alcancemos va a constituir nuestra particular humanidad, sustentada en las redes que establecemos con los otros y que en un sentido antropológico va a dar lugar a nuestra cultura de referencia. Se tiene entonces, que tanto el lenguaje como las redes sociales significativas son insumos ineludibles de la construcción mental y de la subjetividad.

Teniendo en cuenta la anterior, podemos decir por último, que la característica principal del constructivismo aplicable a la psicología en general, y al constructivismo cognitivo en particular, es la aseveración que todo fenómeno cognitivo -desde la percepción y la memoria a la solución de problemas y la conciencia- implica procesos activos y proactivos. En otras palabras, el organismo es un participante activo en su propia experiencia así como en su aprendizaje. Somos constructores de las realidades personales, desde y hacia las cuales respondemos. Más que ser un simple receptor de la experiencia sensorial, el organismo es entendido como un agente activo, anticipatorio y proalimentador (Mahoney, 1991).

Es esta condición del sujeto la que nos abre perspectivas para una psicoterapia efectiva y productiva en el alivio de las "dolencias psíquicas" de nuestros pacientes, (entendido éste como un sujeto que padece) y que, producto de su propia acción de "cura", retoma la esperanza en su porvenir. 


\section{Bibliografía}

BAlBI, J., (1994), Terapia cognitiva posracionalista. Conversaciones con Vittorio Guidano, Buenos Aires, Argentina, Biblos

Beck, A.; Rush, J.; Shou, B.; Emery, G., (1983), Terapia Cognitiva de la Depresión Ed. Desclee de Brouwer, España

Beck, A. Y Freeman, A., (1995), Terapia Cognitiva de los Trastornos de Personalidad, Ed. Paidos, Argentina.

Bowlby, J., (1989), El vínculo afectivo, Barcelona, España, Paidós

Guidano, V (1994), El Sí Mismo en Proceso, Barcelona, Editorial Paidos.

Guidano, V (1987), Complexity of the Self, New York, Guilford Press.

FeIXAs, G., y Miró M. T., (1995), Aproximaciones a la psicoterapia, Barcelona, España, Editorial Piados

KuHN, T., (1971), La estructura de las revoluciones científicas, Fondo de Cultura Económica, México

Kühne, W., (2000), ¿De qué hablan los cognitivos cuando hablan de inconsciente?, en "Memorias de las Primeras Jornadas Clínicas del CAPs" editadas por el Centro de Psicología Aplicada (CAPs) y el Departamento de Psicología de la Universidad de Chile, Santiago de Chile

MAHONEY, M., (1983), Cognición y modificación de conducta, México, Editorial Trillas

Mahoney, M (1988), Cognición y Psicoterapia, Barcelona, Editorial Paidos.

Mahoney, M. (1997) Psicoterapias Cognitivas y Constructivistas, España, Ed. Desclee de Brouwer
Mahoney, (1991), Human Changes Processes: The Scientific Foundations of psychotherapy, New York, Basic Books

Marx, M. H. y Hillix, W. A., (1976), Sistemas y teorías psicológicos contemporáneos, Buenos Aires, Argentina, Editorial Paidós

Maturana, H. y Varela, F. G., (1990), El árbol del conocimiento, Santiago de Chile, Editorial Universitaria Neimeyer, R., y Mahoney, M., (1998), Constructivismo en psicoterapia, Barcelona, España, Editorial Piados

Neimeyer, R.; Mahoney, M (1998), Constructivismo y Psicoterapia , Barcelona, Ed. Paidos.

Piaget, J., (1997), Biología y conocimiento, España, Ed. Siglo Veintiuno

PiAget, J., (1986), El comportamiento motor de la evolución, Argentina, Ed. Nueva Visión.

Popper, K. R. y ECCLES, J. C., (1993), El yo y su cerebro, Barcelona, España, Editorial Labor

Safran, J.; Segal, Z., (1994), El Proceso Interpersonal en la Terapia Cognitiva, Editorial Paidós, Argentina

SeArLe, John. “El Redescubrimiento de la Mente", Ed. Crítica, 1996, España

Skinner, B. F., ([1953] 1981), Ciencia y conducta humana, Barcelona, España, Editorial Fontanella

SKINNER, B. F., (1987), Sobre el conductismo, Buenos Aires, Argentina, Ediciones Orbis.

Sullivan, Harry, (1959), "La Entrevista Psiquiatrica" Editorial Psique, Buenos Aires, Argentina.

Wolman, B. B., (1986), Teorías y sistemas contemporáneos en psicología, Martínez Roca, Barcelona, España. 